\title{
Stochastic Characterization of Flutter using Historical Wind Tunnel Data
}

\author{
Jennifer Heeg ${ }^{1}$ \\ Aeroelasticity Branch, NASA Langley Research Center, Hampton, Virginia, 23681
}

\begin{abstract}
Methods for predicting the onset of flutter during an experiment are traditionally applied treating the data as deterministic values. Uncertainty and variation in the data is often glossed over by using best-fit curves to represent the information. This paper applies stochastic treatments to wind tunnel data obtained for the Piezoelectric Aeroelastic Response Tailoring Investigation model. These methods include modal amplitude tracking, modal frequency tracking and several applications of the flutter margin method. The flutter margin method was developed by Zimmerman and Weissenburger, and extended by Poirel, Dunn and Porter to incorporate uncertainty. Much of the current work follows the future work recommendations of Poirel, Dunn and Porter.
\end{abstract}

\section{Nomenclature}

$\begin{array}{ll}\mathrm{A} & =\text { modal amplitude } \\ \mathrm{F} & =\text { flutter margin } \\ \mathrm{LB} & =\text { lower bound } \\ \mathrm{n} & =\text { number of degrees of freedom (number of experiments) } \\ \boldsymbol{P}(.) & =\text { probability of event occurring } \\ \mathrm{S} & =\text { sample standard deviation } \\ \mathrm{S}^{2} & =\text { sample variance } \\ \mathrm{u} & =\text { value of the random variable associated with a standardized normal distribution function } \\ \mathrm{U} & =\text { standardized Gaussian random variable } \\ \mathrm{UB} & =\text { upper bound } \\ \mathrm{X} & =\text { random variable } \\ \overline{\boldsymbol{X}} & =\text { mean value } \\ \alpha & =\text { significance level } \\ \beta & =\text { modal decay rate, }-\zeta \omega_{n} \\ \gamma & =\text { number of standard deviations from the mean } \\ \kappa & =\text { uncertainty on the mean for a Gaussian process } \\ \sigma & =\text { standard deviation for a Gaussian process } \\ \eta & =\text { shape parameter for a gamma distribution } \\ \lambda & =\text { scale parameter for a gamma distribution } \\ \Theta & =\text { generic random variable } \\ \omega & =\text { modal frequency } \\ \chi & =\text { chi-squared distribution } \\ \zeta & =\text { modal damping }\end{array}$

Subscripted variables:
$\boldsymbol{f}_{\boldsymbol{X}}(\boldsymbol{x})=$ probability density function of the random variable, $\mathrm{X}$
$\mathrm{u}_{\alpha / 2}=$ value of the random variable associated with a standaridized normal distribution function; the probability of a random variable, $\mathrm{U}$, drawn from the standardized normal distribution assuming a value less than $\mathrm{u}_{\alpha / 2}$ is $100 *(1-\alpha / 2) \%$.

\footnotetext{
${ }^{1}$ Research Engineer, Aeroelasticity Branch, Mail Stop 340, AIAA member.
} 
$\beta_{1}, \beta_{2}=$ decay rate of modes 1 and 2

$\sigma_{U B}=$ upper bound on the standard deviation confidence interval

$\omega_{1}, \omega_{2}=$ frequency of modes 1 and 2

$\chi_{n-1, \alpha / 2}^{2}=$ value of the chi-squared distribution where the probability of a random variable being greater than the distribution value is $\alpha / 2$, for $n-1$ degrees of freedom

\section{Introduction}

Z XPERIMENTALLY determining the flutter speed of a flight vehicle or wind tunnel model can be

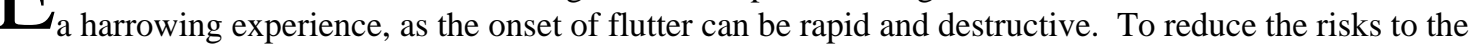
pilot, vehicle, model and facility, many methods of predicting the onset of flutter are used by test engineers. Traditionally, subcritical data and the flutter onset condition have been treated as deterministic values, although the experimental measurements may contain uncertainties due to data acquisition or interpretation and variability due to changes in the conditions or properties. ${ }^{1,2}$

Modal parameter uncertainties and their influence on flutter onset prediction were examined by Poirel, Dunn and Porter in 2005. ${ }^{3}$ They extended the flutter margin method developed by Zimmerman and Weissenburger, ${ }^{4}$ examining the effects of uncertainty in measured modal data. The current work parallels the application of the statistical methods demonstrated by Poirel, Dunn and Porter and attempts to address their recommended areas for future work. These areas include applying the methodology to a wind tunnel model with experimentally determined flutter speed, and exploring the concept of confidence intervals. Additionally, the overall framework they developed for incorporating uncertainties into flutter onset prediction is applied to inverse amplitude tracking. Theoretical stochastic models for the flutter dynamic pressure probability density function are examined, focusing on the Gaussian and gamma distributions. Statistical confidence bounds are computed for the Gaussian model and their implications are discussed.

The current paper distinguishes itself from the prior work by applying the methods to experimental data, where flutter conditions have been measured. Additionally, confidence intervals are determined and their relevance to flutter predictions is assessed.

\section{The Piezoelectric Aeroelastic Response Tailoring Investigation}

This work utilizes data obtained in the Transonic Dynamics Tunnel (TDT) at NASA Langley Research Center in March and November 1994. ${ }^{5}$ The Piezoelectric Aeroelastic Response Tailoring Investigation (PARTI) model was designed as a testbed to demonstrate flutter suppression using piezoelectric actuators. The model was constructed with a graphite-epoxy composite sandwich plate as the primary load-carrying structure. Six fiberglass aerodynamic shell elements were bolted to the plate at single contact points near the leading and trailing edges, and spanned with foam inserts covered with latex. The model is shown in Figure 1, mounted in the TDT test section.

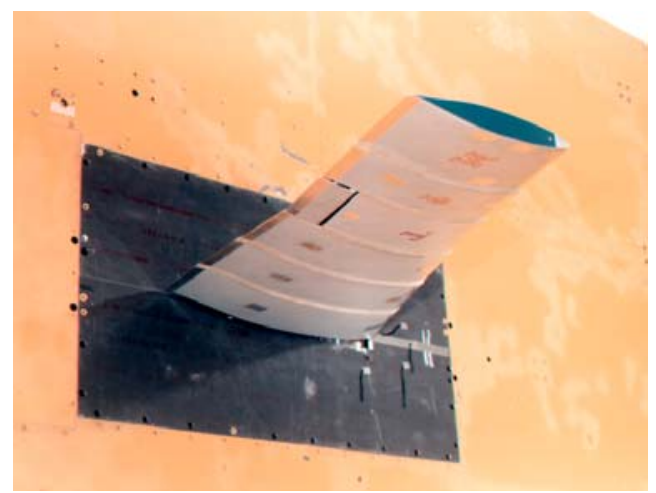

Figure 1. PARTI model mounted in wind tunnel 
Data from testing the PARTI wing lends itself well to stochastic treatment because significant variations in flutter condition and modal properties occurred. The most significant illustration of model change is that the flutter dynamic pressure dropped by $10 \%$ between the March and November tests, Table 1 . A change in the natural frequencies, air off, had also been observed relative to data taken prior to the March test. The repeatability of the aerodynamic shell assembly was questioned, and it was also suspected that the composite plate exhibited creep over the course of the program. These potential sources of variation and change were investigated. Removal and reassembly of the model did not significantly change the natural frequencies or the flutter condition. Non-destructive evaluation of the primary wing structure revealed no damage. It was decided to proceed with the test and treat the differences from the first test to be permanent changes to the model.

Table 1. Flutter characteristics for the two wind tunnel tests

\begin{tabular}{|c|c|c|}
\hline $\begin{array}{c}\text { Flutter Parameters for } \\
\text { Stowed Configuration }\end{array}$ & $\begin{array}{c}\text { First Test } \\
\text { (March) }\end{array}$ & $\begin{array}{c}\text { Second Test } \\
\text { (November) }\end{array}$ \\
\hline Dynamic Pressure (psf) & 85 & 76 \\
\hline Frequency (Hz) & 6.5 & 7 \\
\hline
\end{tabular}

Because the flutter speed had changed so significantly from the prior test, periodic flutter passes were made throughout the test to determine if there were changes due to variability in the tunnel conditions, or fluctuating model properties. Nine experimental flutter points, obtained during the second test, are detailed in Table 2- the flutter points occurred between 73 and 78 psf. All data was obtained using air as the test medium at atmospheric pressure.

Table 2. Measured flutter conditions from November test

\begin{tabular}{|c|c|l|}
\hline Run \# & $\begin{array}{c}\text { Flutter dynamic } \\
\text { pressure (psf) }\end{array}$ & \multicolumn{1}{c|}{ Notes } \\
\hline 2 & $75+$ & $\begin{array}{l}\text { + Tunnel condition was changing as flutter occurred; } \\
\text { dynamic pressure was greater than 75 psf }\end{array}$ \\
\hline 4 & $75+$ & Tunnel condition changing between 75 and 76 psf \\
\hline 20 & 76 & Flutter point at stabilized tunnel condition \\
\hline 24 & 73 & Two passes, both flutter at 73 psf stabilized condition \\
\hline 28 & 74 & Two passes, both flutter at 74 psf stabilized condition \\
\hline 35 & $75+$ & Tunnel condition changing between 75 and 76 psf \\
\hline 36 & 78 & With trip strips \\
\hline
\end{tabular}

Although the flutter condition did not repeat exactly during each of these runs, the data indicated that a permanent change in the model had occurred. This required acquisition of new model identification data, and redesign of the control laws. This effort produced a large data set, with the engineers particularly focused on observing variation in the modal and flutter characteristics. The remainder of this paper focuses on open loop data acquired during the November test, and treating the data from a statistical standpoint.

\section{Inverse Amplitude Tracking Method}

\section{A. On-line prediction}

The procedure for conducting a flutter pass during the PARTI testing was to start at a subcritical dynamic pressure and acquire data. The data was analyzed in near real time to produce modal amplitudes, frequencies and dampings. On-line flutter onset predictions were updated and the dynamic pressure increased. This was repeated until flutter was encountered.

One on-line method for predicting flutter onset is to track and curve fit the inverse of the modal amplitudes. In the PARTI test, data was acquired from accelerometers; a Fourier transform was applied to the data. Peaks appearing in the data correspond to the response at the aeroelastic frequencies of the system. The 
peak value at each of the aeroelastic modal frequencies was inverted and plotted. Flutter can be indicated by extremely large modal amplitudes or, correspondingly, it can be indicated by near-zero inverse modal amplitudes. Figure 2 shows a data set obtained from a single flutter pass.

The inverse of each amplitude for the first two aeroelastic modes is plotted versus dynamic pressure. Flutter onset predictions were generated by linear fits to each mode and extrapolated to the x-axis crossing. Throughout the test, it was found that the first mode's amplitude was the most reliable for flutter prediction; this is illustrated by the on-line prediction results presented in Table 3. The predictions shown in the table were generated by linear fits using all of the data at and below the corresponding dynamic pressure. The experimentally determined flutter condition for this run was between 75 and 76 psf. Using all of the data up to 75 psf produces a slightly non-conservative flutter prediction, $76.2 \mathrm{psf}$.

Examination of mode 2 illustrates an important point regarding the use of the inverse amplitude tracking method- engineers rarely rely on an automated linear fit to the full data set. The data at $30 \mathrm{psf}$ skews the fit to data that is otherwise very linear. Typically, the engineer monitoring the process would ignore the $30 \mathrm{psf}$ data point and provide a flutter estimate based on a modified linear fit. The revised prediction, however, is still non-conservative.

The remainder of the discussion of the inverse amplitude tracking method will use only data corresponding to the first aeroelastic mode.

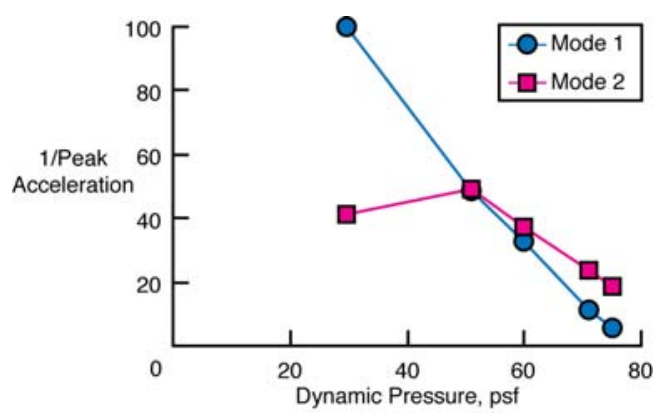

Figure 2. On-line flutter prediction, inverse amplitude tracking, Run 2

Table 3. On-line flutter predictions, Run 2

\begin{tabular}{|c|c|c|c|c|}
\hline & \multicolumn{2}{|c|}{ Mode 1 } & \multicolumn{2}{c|}{ Mode 2 } \\
\hline $\begin{array}{c}\text { Dynamic } \\
\text { Pressure } \\
\text { (psf) }\end{array}$ & 1/amplitude & $\begin{array}{c}\text { Flutter } \\
\text { prediction } \\
\text { (psf) }\end{array}$ & $\begin{array}{c}\text { Flutter } \\
\text { prediction } \\
\text { (psf) }\end{array}$ \\
\hline 29.75 & 100.0 & & 41.0 & \\
51 & 48.5 & 71.0 & 49.3 & -75.5 \\
60 & 32.6 & 73.5 & 37.5 & 1704.7 \\
71 & 11.1 & 75.1 & 23.6 & 150.6 \\
75 & 5.6 & 76.2 & 18.9 & 121.8 \\
\hline
\end{tabular}

\section{B. Stochastic post-test data analysis: inverse amplitude histogram method}

While the inverse amplitude method described above worked well for the PARTI test, the data for each run had to be used in isolation. When multiple runs were compared, it was observed that there was significant scatter in the data. Normalized inverse amplitude data sets from eight flutter clearance passes are presented in Figure 3, illustrating this scatter. 


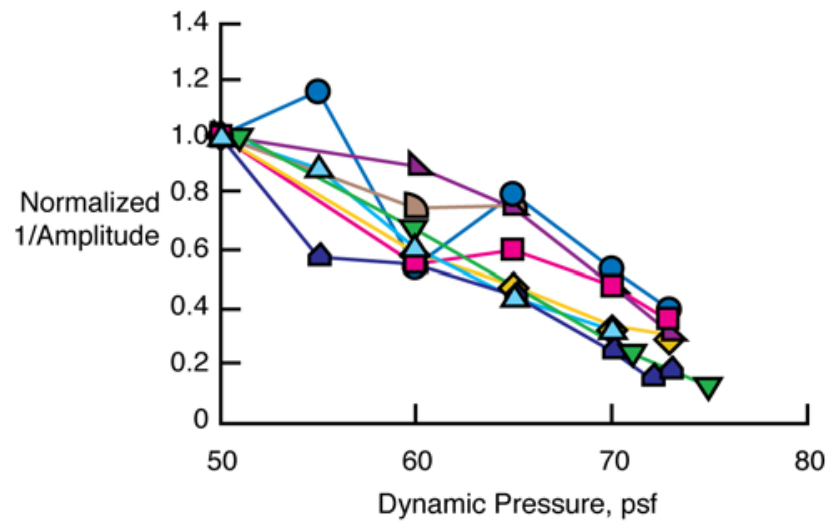

Figure 3. Inverse amplitude tracking method for flutter clearance

With all of the data from the test in hand, a stochastic treatment for the inverse amplitude data was developed and applied in a manner similar to the on-line prediction technique. Choosing one measured value of the amplitude at each of the test conditions, a linear fit was performed to determine a flutter onset prediction. This process was repeated, exhaustively choosing new values of amplitude at each of the test conditions and performing linear fits and predictions. The number of flutter dynamic pressure predictions is the number of degrees of freedom in this experiment.

A normalized histogram of the flutter onset predictions is shown in Figure 4. The histogram approximates a probability density function, (PDF). Statistics of the process were calculated, yielding a mean of 83.9 psf and a standard deviation of $3.97 \mathrm{psf}$. The median value, influenced by the presence of a right-side tail is 83.34 psf. The peak indicates the statistical mode value, $82 \mathrm{psf}$. The distribution is reasonably approximated as Gaussian; this idealized distribution is also shown in Figure 4. The cumulative distribution function, (CDF), Figure 5, is obtained by integrating the area under the PDF. The graph shows the probability that flutter has not occurred at a dynamic pressure lower than a given $\mathrm{x}$-axis value.

Stochastic treatment of the amplitude data produces a non-conservative prediction of the flutter dynamic pressure. There are two ways to observe this from the data. The experimental CDF, Figure 5, shows the probability that flutter will occur below a given dynamic pressure. The percent probability that flutter will not occur below a given dynamic pressure is determined by subtracting the vertical axis value from $100 \%$. The value of 5\% probability shown on the vertical axis corresponds to $78 \mathrm{psf}$ on the horizontal axis. Therefore, there is a $95 \%$ probability that the model has not fluttered at 75 psf.

The second method of observing the flutter prediction is to consider the Gaussian model of the process. Using the statistics to provide 95\% probability that the system has not fluttered requires that the lower bound be calculated by subtracting twice the standard deviation from the mean. This gives a lower bound of 76 psf as the flutter dynamic pressure. It is not conservative to say that $95 \%$ of the time, the system will not flutter at conditions below this- recall from Table 2 that out of all nine points where flutter occurred, only one fluttered above this condition.

Poirel, Dunn and Porter suggest using the mode as the central statistic to induce conservatism into the process. Applying twice the standard deviation to the mode gives a 95\% confidence lower flutter bound of 74 psf, which is more conservative. 


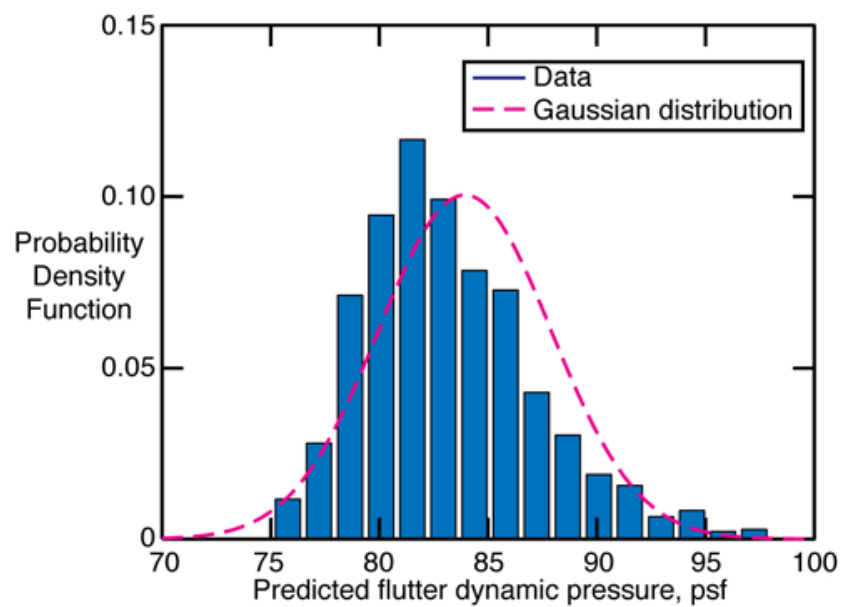

Figure 4. Histogram of flutter predictions, using inverse amplitude tracking

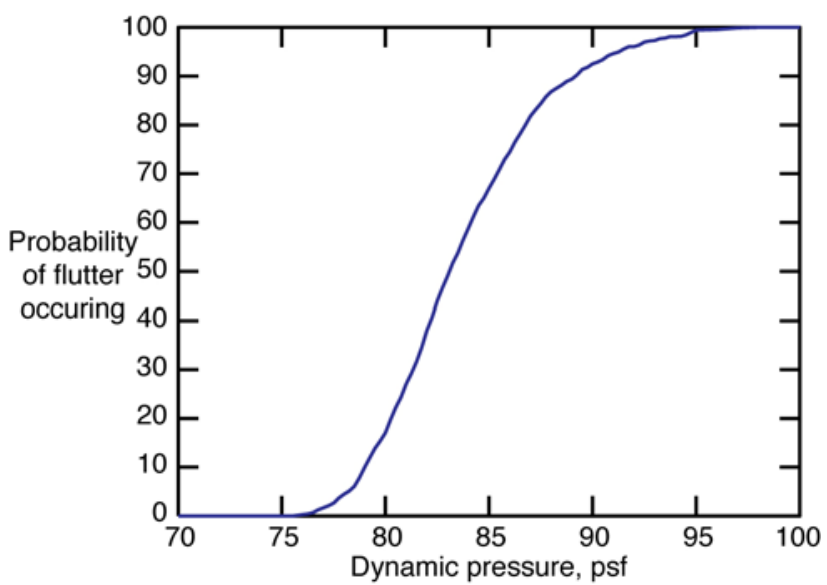

Figure 5. Cumulative distribution function, inverse amplitude method, linear fit

Statistical treatment of the inverse amplitude data produced results which were not conservative. There are two primary causes: 1) A linear fit,constrained to use randomly combined data at each subcritical condition, did not generally give a conservative flutter prediction; engineering judgment generally needs to be applied to inverse amplitude data to accurately assess the flutter condition; 2) A Gaussian distribution can not fit the data accurately due to the skewness of the distribution of the flutter dynamic pressures.

\section{Frequency Monitoring}

Modal frequency tracking was used as a qualitative guide for predicting the flutter condition during the PARTI test. Flutter onset was predicted by visual extrapolation of the frequencies to their coalescence. Knowledge of the analytical trends or the behavior on previous flutter passes was essential to accurately using this information. On-line data acquired for monitoring the frequencies of the first three aeroelastic modes is shown in Figure 6. This data corresponds to the amplitude data shown previously in Figure 3.

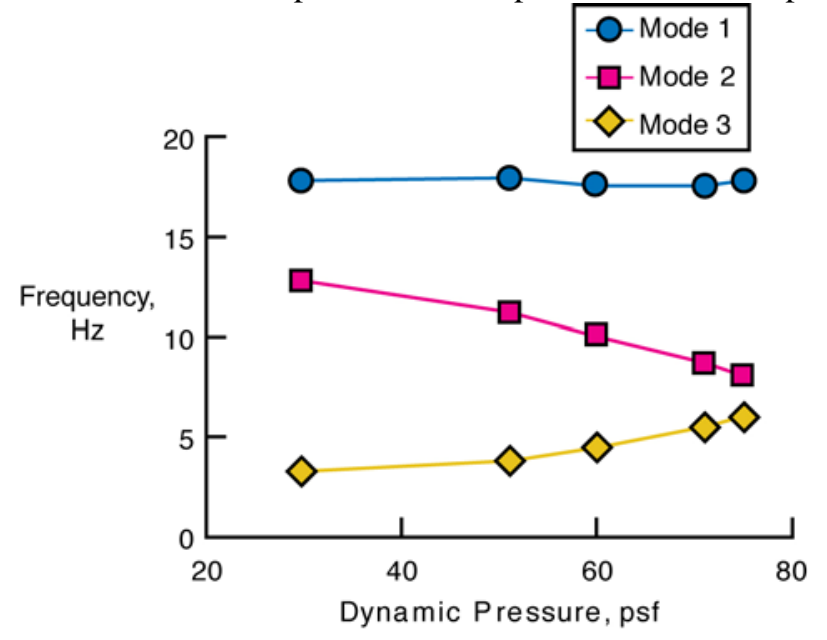

Figure 6. Flutter clearance run, on-line data analysis tracking the frequencies of the first three modes

This qualitative method can not be adapted to use stochastic techniques, but provides the data essential for using the flutter margin method. All of the natural frequency measurements obtained during the test for the first 2 modes are shown in Figure 7, illustrating a significant amount of scatter in the measurements. 


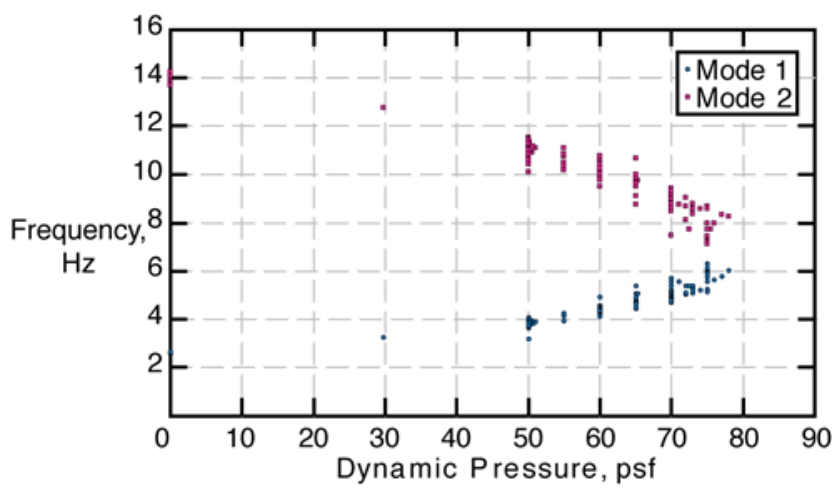

Figure 7. Scatter in modal frequency measurements

Histograms of the frequency information acquired at $50 \mathrm{psf}$ are presented in Figure 8. Although neither distribution is precisely Gaussian, the statistics of the process were computed using this assumption. The mean, variance, mode and median values for each of the modal frequencies are given in Table 4.

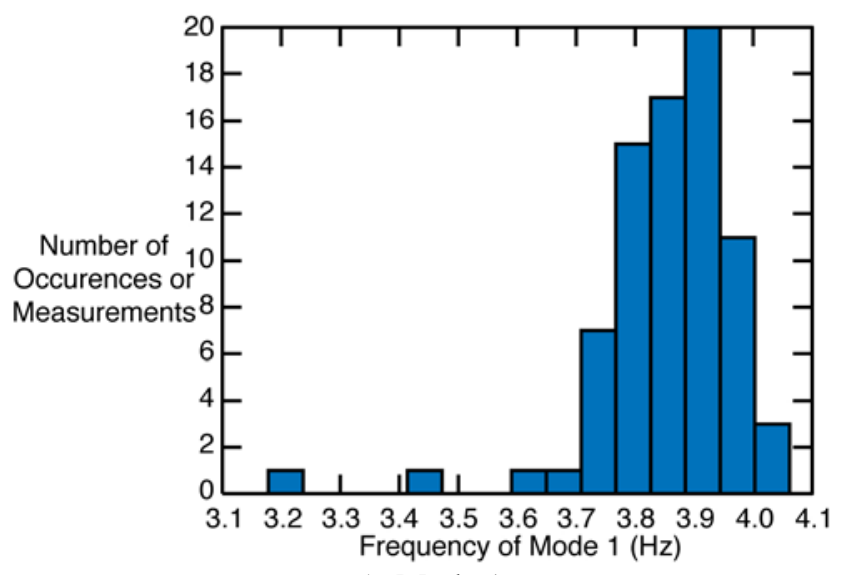

a) Mode 1

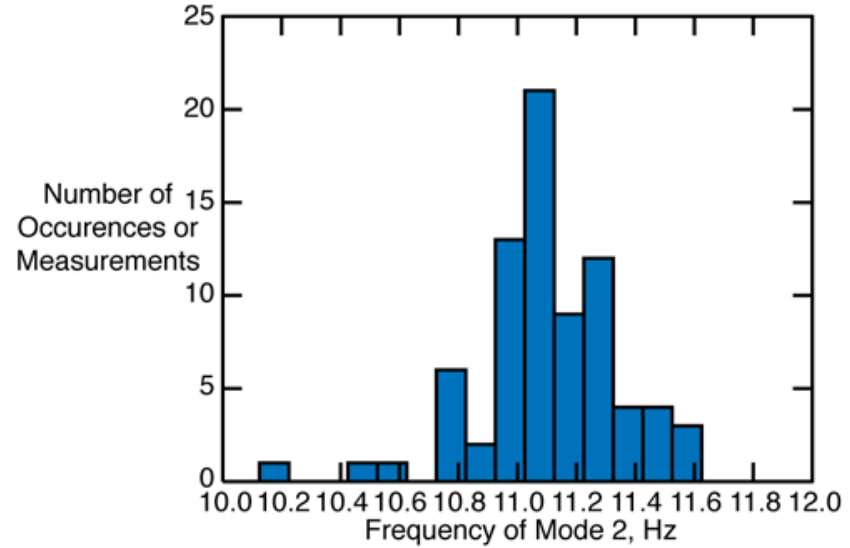

b) Mode 2

Figure 8. Frequency histograms at 50 psf

Table 4. Statistical Properties of Frequencies at 50 psf

\begin{tabular}{|l|l|l|l|l|}
\hline & & Mean & Variance & Median \\
\hline \multirow{2}{*}{$\omega_{\mathrm{n}}$} & Mode 1 & 3.868 & 0.017 & 3.875 \\
\cline { 2 - 5 } & Mode 2 & 11.107 & 0.059 & 11.0125 \\
\hline
\end{tabular}

The statistics were computed for the natural frequencies at each of the test conditions from 50 to 75 psf. Using the mean and the standard deviation, 95\% probability values $(+/-2 \sigma)$ on the frequencies were computed and are shown in Figure 9. This figure provides an interpretation of the potential variation in the flutter dynamic pressure. Using the $95 \%$ probability values, and assuming the worst-case flutter scenario, the dashed lines nearest each other represent the frequency characteristics of the aeroelastic system. Using these trends, flutter is imminent at 75 psf. However, if the least conservative case is assumed, the outer dashed lines represent the frequency characteristics of the aeroelastic system. While they have started to migrate towards each other, it is not evident that the onset of flutter will occur prior to $80 \mathrm{psf}$.

The consequences of assuming that the Gaussian statistics accurately represent the experimental data can be seen in Figure 9b. Here, the data from Figure 7, which shows the experimental variation in the frequencies, has been overlaid with the Gaussian 95\% probability bounds. Where the experimental data points lie outside the bounds, it is obvious that the Gaussian representation is not accurate- representing the frequency data by the statistics of a Gaussian process is not a good methodology to employ. 


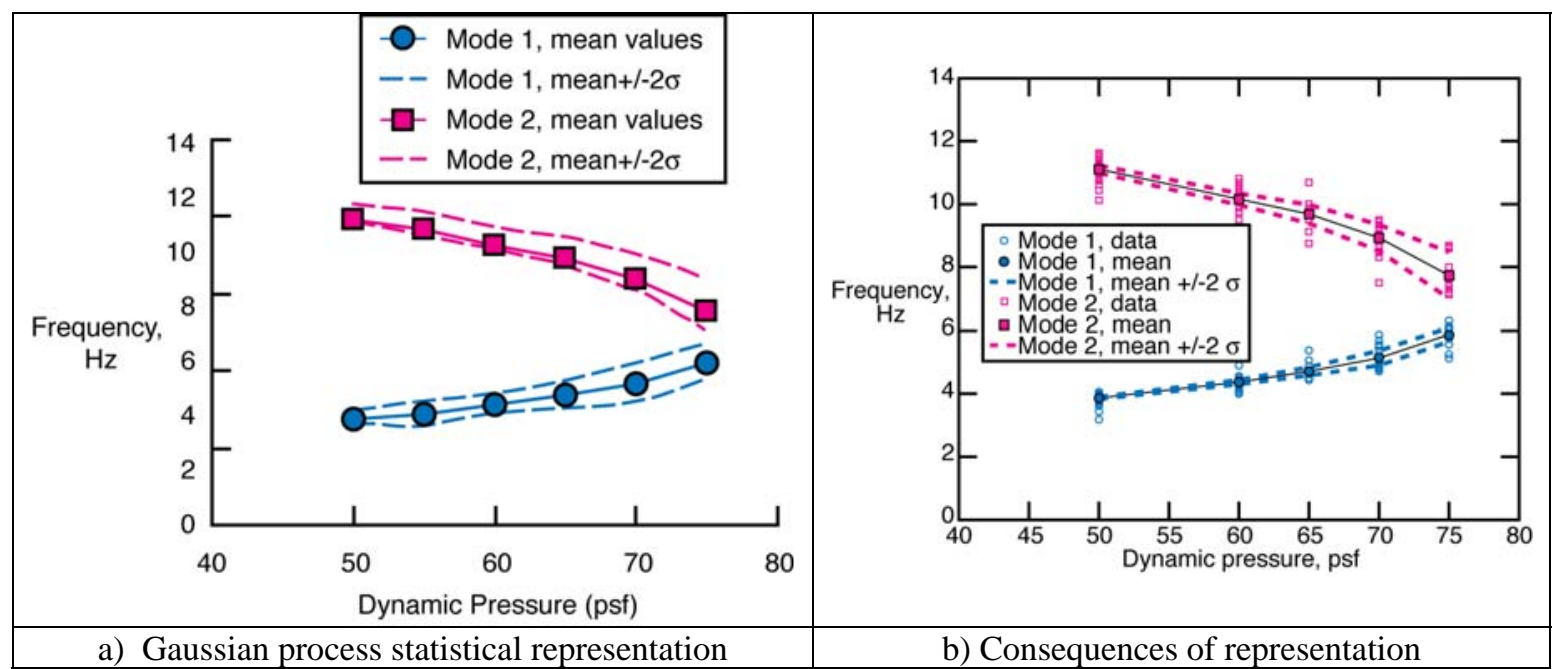

Figure 9. Statistical calculations of frequency versus test condition

\section{Flutter Margin Method}

Zimmerman and Weissenburger ${ }^{4}$ defined a flutter stability parameter which they demonstrated to be highly effective at predicting flutter onset for classical bending-torsion flutter. Their method was developed based on an idealized bending-torsion system subject to quasi-steady aerodynamics. They developed a parameter that they termed the flutter margin, which incorporated measured frequencies and decay rates at a given dynamic pressure. Using the flutter margin at a series of dynamic pressures, they extrapolated to find the value of dynamic pressure that had a flutter margin of 0 , and thus produced a prediction of the flutter dynamic pressure. They also showed that the flutter margin was a second order function of dynamic pressure. At a given test condition, the flutter margin is calculated using Equation 1.

$$
\begin{aligned}
& F=\left[\left(\frac{\omega_{2}^{2}-\omega_{1}^{2}}{2}\right)+\left(\frac{\beta_{2}^{2}-\beta_{1}^{2}}{2}\right)\right]^{2}+4 \beta_{1} \beta_{1}\left[\left(\frac{\omega_{2}^{2}+\omega_{1}^{2}}{2}\right)+2\left(\frac{\beta_{2}+\beta_{1}}{2}\right)^{2}\right] \\
& -\left[\left(\frac{\beta_{2}-\beta_{1}}{\beta_{2}+\beta_{1}}\right)\left(\frac{\omega_{2}^{2}-\omega_{1}^{2}}{2}\right)+\left(\frac{\beta_{2}+\beta_{1}}{2}\right)^{2}\right]^{2}
\end{aligned}
$$

Equation 1

Poirel, Dunn and Porter recognized that the flutter margin method was sensitive to scatter in the measured values of the frequencies and the decay rates. They applied the flutter margin method in a manner that captured uncertainties in the measurements.

They utilized two basic methods to generate flutter predictions: 1) A of values for the modal parameters at each test condition was used to compute the flutter margin; the mean value of flutter margin at each dynamic pressure was used to extrapolate to the flutter prediction; 2) The range of values for the modal parameters at each test condition was used to compute all possible values of flutter margin; all possible combinations of flutter margin at each of the test conditions were then used to produce an extrapolated flutter prediction. An identical application of these methods follows, using measurements from the PARTI experiment. 


\section{A. Mean flutter margin method}

The first method requires determining the mean value of the flutter margin at each dynamic pressure.

These mean values are then plotted as a function of dynamic pressure and a quadratic curve fit is applied.

The extrapolation of this curve fit to 0 produces the estimate of the flutter dynamic pressure.

As previously shown, numerous measurements of frequency were obtained at each of the test conditions. Modal damping was measured only sporadically during the PARTI test. Fortunately, Poirel, Dunn and Porter determined that the damping variation has much less impact on the flutter margin calculations than the frequency variation. Thus, the mean values of the measured modal damping at each dynamic pressure were used in computing flutter margins.

For each test condition, all of the combinations of measured frequencies at that condition were used to compute values of flutter margin. This gave rise to a large number of possible flutter margin values. The mean of these values at each test condition was then curve fit and a flutter prediction was extrapolated. The results are shown in Figure 10. The fit produces a very good estimate of the flutter condition. This is true even when only the lowest 2 dynamic pressures are used in the curve fit.

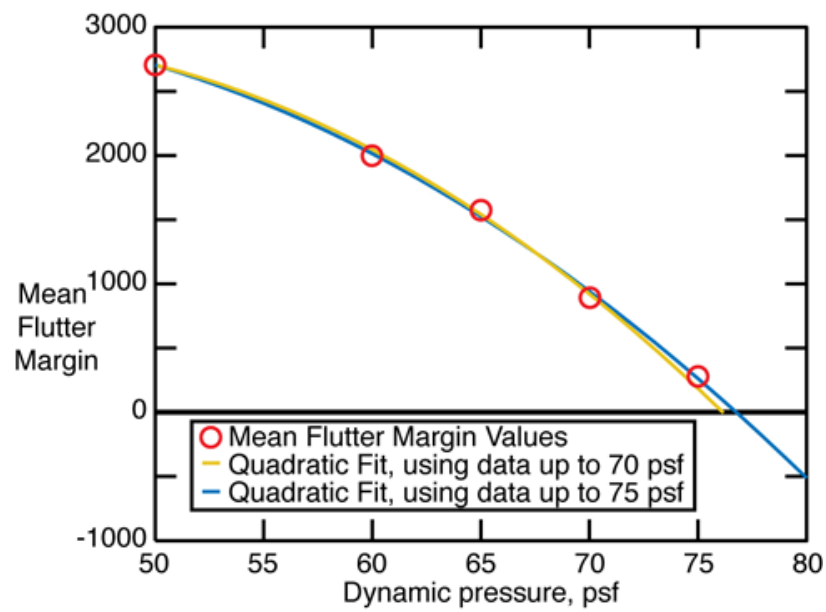

Figure 10. Mean flutter margin extrapolation results

\section{B. Flutter margin histogram method}

The flutter margin histogram procedure is similar to the inverse amplitude histogram procedure previously described. Applying the second method begins the same as the first method. All possible values of flutter margin at each condition were generated, exhaustively using the measured frequencies. The damping at each test condition was taken to be the mean measured value. Example histograms of the flutter margin results are shown in Figure 11. Figure 11a shows all calculated flutter margin values at 50 psf; Figure 11b shows all values for 70 psf.

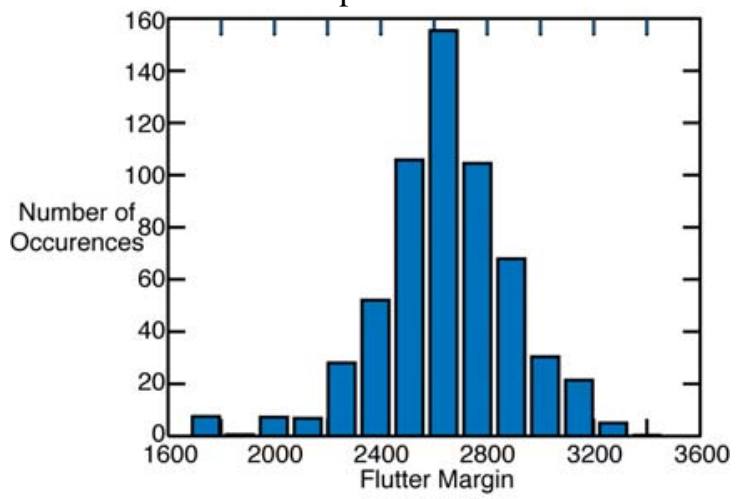

a) $50 \mathrm{psf}$

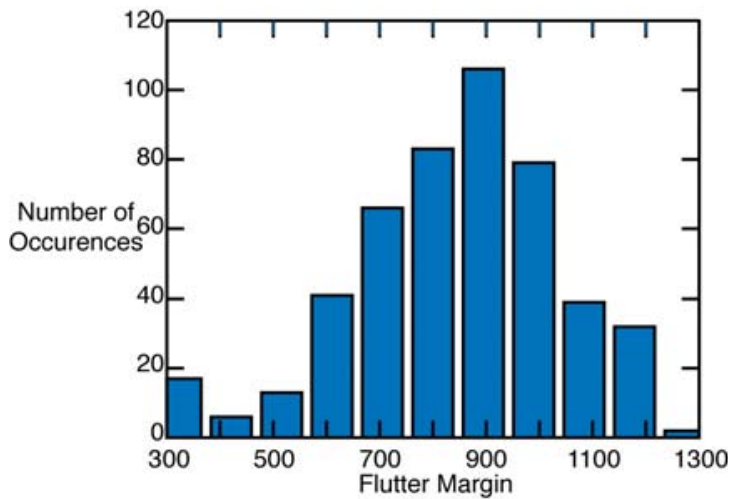

b) $70 \mathrm{psf}$

Figure 11. Histogram of flutter margin at example test conditions 
At this point, the second method deviates from the first. At each of the test conditions, there are large numbers of values for the flutter margin. In this analysis, one value of the flutter margin was selected at each of the test conditions. The value of flutter margin at each test condition was selected by random draw from all of the calculated values. A quadratic polynomial was fit to these flutter margin values, as a function of dynamic pressure to produce a prediction of flutter dynamic pressure. This process was repeated to produce 100,243 possible flutter values. The number of flutter dynamic pressure values is the number of degrees of freedom in this experiment. A normalized histogram of the results is shown in Figure 12.

Identical processes were applied to the data, using only information available up to $65 \mathrm{psf}$ and then up to 70 psf. The statistics of these processes are given in Table 5 . Of the three central statistics, the mode is always the lowest value. The mean and variance values vary substantially as more test conditions are included in the analysis. As data is included closer to the flutter condition, the variance in the predictions decreases. This is expected, because the subcritical data at $75 \mathrm{psf}$ indicates that flutter will occur imminently. However, including subcritical data at 75 psf all but precludes consideration that flutter could already have occurred. Recall from the flutter points discussed earlier that numerous flutter points did occur below 75 psf.

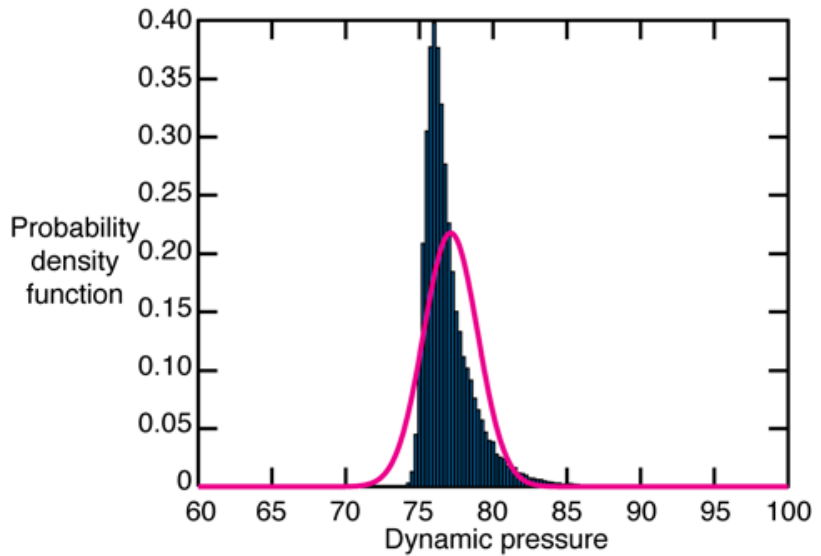

Figure 12. Histogram of flutter dynamic pressure predictions, Flutter margin histogram method

Table 5. Statistics of predicted flutter dynamic pressures (psf)

\begin{tabular}{|c|c|c|c|c|}
\hline Data Included & Mean & Variance & Median & Mode \\
\hline $50-65 \mathrm{psf}$ & 114.3 & $1.2 \mathrm{e} 7$ & 69.9 & 66.5 \\
\hline $50-70 \mathrm{psf}$ & 76.9 & 17.5 & 75.8 & 74.2 \\
\hline $50-75 \mathrm{psf}$ & 77.1 & 3.4 & 76.6 & 76.0 \\
\hline
\end{tabular}

\section{Fitting a Theoretical Model to the Flutter Dynamic Pressure}

Calculating the mean and variance assumes an underlying theoretical Gaussian model of the distribution. A Gaussian process has symmetric distribution about the mean, with the sharpness of the peak defined by the variance. Using the estimates for the mean and the variance, the theoretical Gaussian distribution is plotted with the flutter dynamic pressure histogram in Figure 12. It shows the shortcomings of this model; the Gaussian process captures neither the peak nor the skewness of the distribution.

Non-symmetric distributions have been used for modeling this type of process. A gamma distribution resembles the shape of the flutter dynamic pressure histogram. However, obtaining a reasonable fit to the data required that a threshold value or a bias be applied in order to avoid numerical issues associated with calculating the gamma function for large values of shape parameter. A maximum likelihood, (MLE), parameter estimation method ${ }^{6}$ for the gamma distribution parameters ${ }^{7}$ was programmed. Using the biased 
data, maximum likelihood estimates of the shape and scale parameters were 6.971 and 1.673, respectively.

The plot in Figure 13 shows the histogram of the biased data, using the solid blue line with circles. The MLE gamma function is shown by the dashed cyan line. Note that the peak value is approximately $75 \%$ of the peak value from the histogram of flutter dynamic pressure. It is also offset from the peak, and is broader than the histogram. A second gamma distribution, which was manually generated and is not optimized in any sense other than appearance, is shown by the solid magenta line. The shape parameter is 9.0 ; the scale parameter is 2.7 . The peak value is much closer to that of the histogram, but note how the distribution has a left shift. This curve will give a more conservative estimate of the flutter dynamic pressure than the MLE result.

\begin{tabular}{|lccc|} 
& $\begin{array}{c}\text { Shape } \\
\text { Parameter }\end{array}$ & $\begin{array}{c}\text { Scale } \\
\text { Parameter }\end{array}$ & $\begin{array}{c}\text { Generated } \\
\text { through }\end{array}$ \\
& $\eta$ & $\lambda$ & \\
\hline - - Gamma Distribution & 6.971 & 1.673 & Maximum Likelihood \\
- Gamma Distribution & 9.0 & 2.7 & Manual Fit \\
$\Theta$ Histogram of Data & & & \\
\hline
\end{tabular}

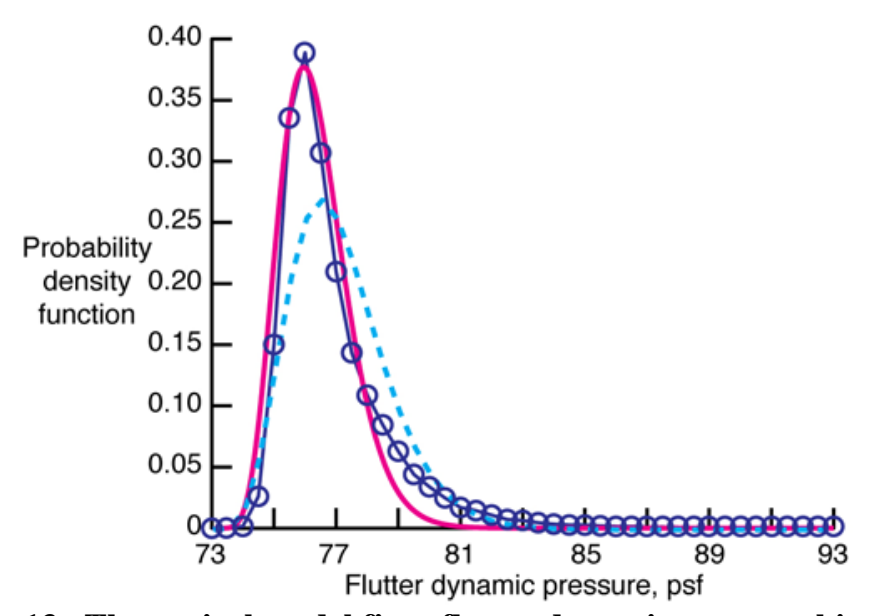

Figure 13. Theoretical model fit to flutter dynamic pressure histogram

A further test of the goodness of a theoretical model is provided by examining a quantile-quantile plot. From the NIST/SEMATECH e-Handbook of Statistical Methods ${ }^{8}$, "The quantile-quantile (q-q) plot is a graphical technique for determining if two data sets come from populations with a common distribution. A q-q plot is a plot of the quantiles of the first data set against the quantiles of the second data set. By a quantile, we mean the fraction (or percent) of points below the given value. That is, the 0.3 (or 30\%) quantile is the point at which $30 \%$ percent of the data fall below and $70 \%$ fall above that value.”

A q-q plot is presented in Figure 14, where there experimental data is compared to itself, the experimental data is compared to the maximum likelihood estimate of the gamma distribution, and the experimental data is compared to the manual fit gamma distribution. The experimental data compared to itself shows what the data would look like if one of the theoretical models were an exact fit to the data.

When a quantile plot goes above the exact fit line, the theoretical model is non-conservative. The black dashed lines in Figure 14 give examples for the two theoretical distributions. From the vertical axis, the horizontal line at 0.6 intersects the maximum likelihood distribution at a point corresponding to 0.5 . This says that $60 \%$ of the data points, or values for flutter dynamic pressure, are below the dynamic pressure where the model estimates that only $50 \%$ of them lie below. Thus, the maximum likelihood model is not conservative, even though the overall fit to the data appears better than the manual fit.

When the quantile plot goes below the exact fit line, the theoretical model is conservative, as in the case of the manual fit gamma distribution. From the vertical axis, the horizontal line at 0.35 intersects the manual fit distribution at a point corresponding to 0.5 . This says that $35 \%$ of the data points, or values for flutter 
dynamic pressure, are below the dynamic pressure where the model estimates that $50 \%$ of the values would lie below. Thus, the manual fit model gives a conservative estimate of the flutter dynamic pressure distribution.
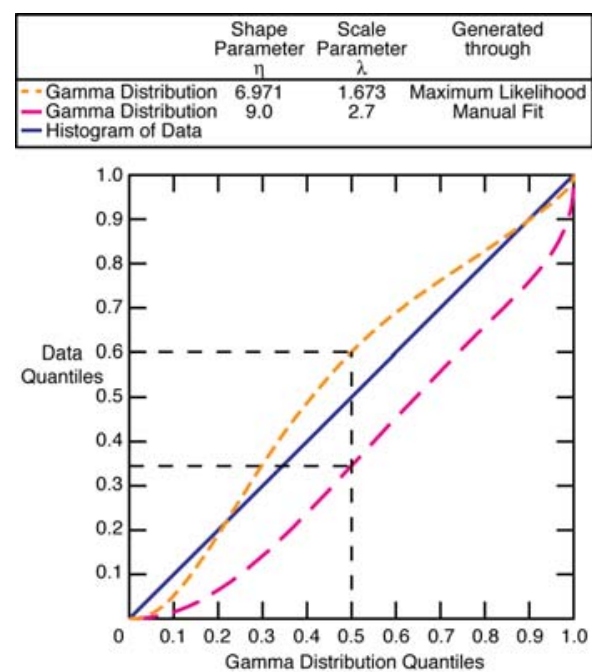

Figure 14. Quantile plot using gamma distributions

\section{Probability levels associated with flutter}

The quadratic curve fits to the flutter margin data produce some cases which do not flutter. Table 6 shows the results for the three ranges of test conditions which were considered. Using only data up to 65 psf, only $38.5 \%$ of the curve fits indicated that the system would flutter. As more data was included, the probability of flutter occurring rose to $99.1 \%$. While this indicates that including higher dynamic pressure information in the subcritical data set produces a better prediction of flutter, closer scrutiny is required.

The data was also examined to determine the dynamic pressure bound corresponding to 95\% probability of being flutter-free. This was done for each of the three ranges of test condition, corresponding to the three data rows in Table 6 . The $3^{\text {rd }}$ column of Table 6 shows the results obtained when the information in the experimental CDF is used. As more data is incorporated, the dynamic pressure bound increases. It is interesting to note that each row of data indicates that the flutter dynamic pressure is very close to the highest condition considered.

The final two columns of the table give the dynamic pressures that correspond to 95\% probability of being flutter-free if the statistics of the estimated Gaussian processes are used. The high variance using the first range of test conditions produces a negative dynamic pressure lower bound. The second data set considers conditions in the subcritical data set that lie strictly below the lowest obtained experimental dynamic pressure. The results produce conservative estimates for the bound on flutter: $68.5 \mathrm{psf}$ using the mean value and $65.9 \mathrm{psf}$ using the mode value. The third data set, considering all subcritical data available, gives higher values of the $95 \%$ probability lower bound.

Table 6. Probabilities associated with flutter conditions

\begin{tabular}{|c|c|c|c|c|}
\hline & & \multicolumn{3}{|c|}{$\begin{array}{c}\text { Dynamic pressure corresponding to } \\
\text { 95\% probability of being flutter-free, (psf) }\end{array}$} \\
\hline $\begin{array}{c}\text { Test conditions } \\
\text { included }\end{array}$ & $\begin{array}{c}\text { Probability that } \\
\text { flutter will occur, } \\
\text { (\% of cases that } \\
\text { fluttered) }\end{array}$ & $\begin{array}{c}\text { Using CDF } \\
\text { data directly }\end{array}$ & $\begin{array}{c}\text { Based on } \\
\text { Gaussian } \\
\text { process, } \\
\text { Mean - } 2\end{array}$ & Mode $-2 \sigma$ \\
\hline $50-65$ psf & 38.5 & 66.5 & N/A & N/A \\
\hline $50-70$ psf & 90.2 & 72.4 & 68.5 & 65.9 \\
\hline $50-75$ psf & 99.1 & 75.3 & 76.0 & 72.3 \\
\hline
\end{tabular}


Another way to examine the probability, is to determine dynamic pressures corresponding to different probability levels for flutter occurrence. This has been done using the gamma distributions, and using all subcritical data available. The CDFs of the data and the gamma distributions are shown in Figure 15. Example calculations for the conditions corresponding to $95 \%$ probability of being flutter-free are illustrated in the close-up, part $b$ of this figure.

The dashed line originating on the vertical axis shows when the CDFs indicate that there is only a 5\% probability of flutter occurring. This corresponds to a 95\% probability of being flutter-free. The first CDF that this line intersects is the manual fit gamma distribution, 74.7 psf. Next, it intersects the maximum likelihood gamma distribution at 74.9 psf. Finally, it intersects the CDF produced directly by the quadratic curve fits to the data, $75.3 \mathrm{psf}$. This progression indicates that both gamma distribution models produce conservative values for the $95 \%$ flutter-free estimates.
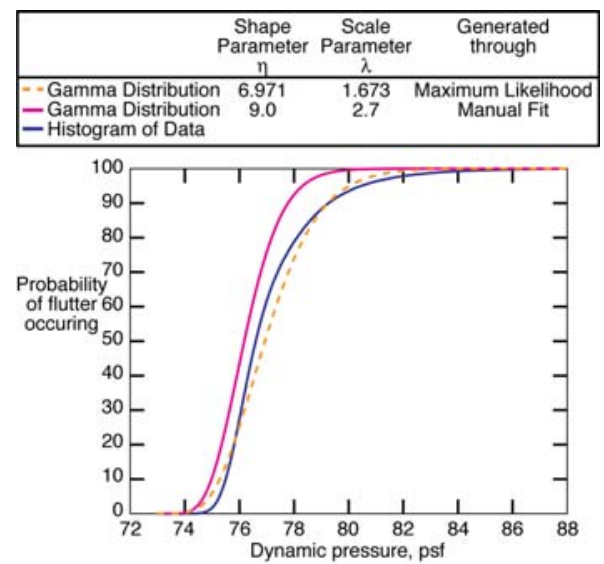

a) Showing full range of possible flutter conditions

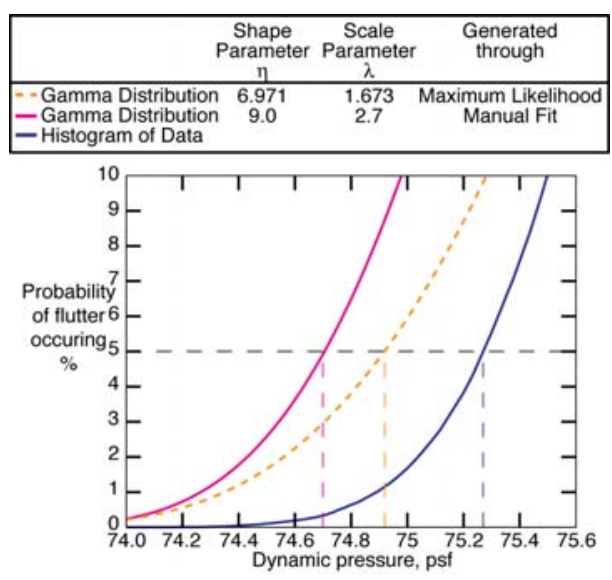

b) $95 \%$ Probabability conditions

Figure 15. Cumulative distribution functions for the gamma distributions

However, reviewing the quantile plot, it is apparent that this will not be true for the maximum likelihood model at other probability levels. In many statistical analyses, it is customary to show data corresponding to different $\sigma$-levels. Although these are strictly applicable only to a Gaussian process, these levels correspond to levels of probability. Using $1 / 2-, 1-, 2$ - and 3- $\sigma$ levels, Table 7 summarizes the dynamic pressures corresponding to the associated levels of probability. The columns show the results for direct use of the flutter margin data CDF, the Gaussian model and the two gamma distributions.

Notice that for each probability level, even though the Gaussian model is the poorest representation of the data, the symmetric properties of the distribution allow it to produce the most conservative values for flutter onset.

Table 7. Flutter predictions corresponding to probability levels, using data up to 75 psf

\begin{tabular}{|c|c|c|c|c|c|}
\hline & & \multicolumn{4}{|c|}{ Dynamic Pressure (psf) } \\
\hline $\begin{array}{c}\text { Probability of } \\
\text { being flutter- } \\
\text { free } \\
(\%)\end{array}$ & $\begin{array}{c}\text { Sigma level } \\
\text { of a Gaussian } \\
\text { distribution, } \\
\text { (for reference } \\
\text { only) }\end{array}$ & $\begin{array}{c}\text { Flutter margin } \\
\text { histogram } \\
\text { results, directly }\end{array}$ & $\begin{array}{c}\text { Gaussian, } \\
\text { point estimates }\end{array}$ & $\begin{array}{c}\text { Gamma, } \\
\text { maximum } \\
\text { likelihood fit }\end{array}$ & $\begin{array}{c}\text { Gamma, } \\
\text { manual fit }\end{array}$ \\
\hline 38 & $1 / 2$ & 77.02 & 76.21 & 77.42 & 76.5 \\
\hline 68 & 1 & 76.11 & 75.29 & 76.28 & 75.70 \\
\hline 95 & 2 & 75.27 & 73.46 & 74.92 & 74.70 \\
\hline 99.7 & 3 & 74.68 & 71.63 & 74.06 & 74.04 \\
\hline
\end{tabular}


Also note that the maximum likelihood method for determining the shape and scale parameters did not build conservatism into the model, except at the very high and very low probability of flutter occurring. Manual adjustment of the parameters allowed this conservatism to happen. In a case like this, where conservatism is essential, a different fit criterion might be a better choice. The gamma distribution may provide the best fit that can be achieved with a 3-parameter model, where the three parameters are the shape, scale and threshold. Higher order distributions, as suggested by Pimentel et al, ${ }^{9}$ Bandourian et al, ${ }^{10}$ and Clark et al. ${ }^{11}$ would likely provide better fits to the data.

\section{Confidence Intervals}

The threshold gamma distribution model fits the flutter margin data much better than the Gaussian distribution model. However, the equations for calculating the confidence intervals for a gamma distribution are not widely-published. For the first cut at determining confidence levels for the flutter dynamic pressure, it was assumed that the distribution could be estimated as a Gaussian process. Methods for determining confidence intervals for Gaussian processes are well known and easily accessible. ${ }^{6,8,12,13}$

There are several approaches to estimating statistical parameters from a set of sample measurements. The first approach generates a single value to estimate the parameter. Here, the sample mean has been calculated as an estimate of the process mean and the sample variance has been calculated as an estimate of the process variance.

A second approach to estimating a parameter is interval estimation, which produces bounds on the parameter value. These bounds give numerical information for the parameter and also indicate the level of confidence that can be placed on the numerical value based on the samples. The confidence interval on the mean value and on the variance can be computed using the normal distribution function and the chi-squared distribution function, respectively. Upper and lower limits of an interval are defined such that, with a specified level of confidence, the true parameter will lie in this interval. The interval (LB,UB) is the 100(1$\alpha) \%$ confidence interval for a parameter $\Theta,{ }^{6}$ as expressed in Equation 2.

$$
P(L B<\Theta<U B)=1-\alpha
$$

Equation 2

In evaluating the flutter points, the goal is to determine the lowest value at which flutter is likely to occur with a given probability. Thus, a lower bound on the flutter dynamic pressure is calculated using the general form of Equation 3.

$$
L B=\bar{X}-\kappa-\gamma^{*} \sigma_{U B}
$$

Equation 3

A graphical illustration applying these confidence intervals is illustrated in Figure 16. The plot shows a standardized Gaussian distribution probability density function, $\mathrm{f}_{\mathrm{X}}(\mathrm{x})$ with a solid blue line. The solid red line at $\mathrm{X}=0$ shows the mean value, $\overline{\boldsymbol{X}}$, of the process. The dashed red line, just to its left indicates the uncertainty associated with the mean of the process, $\kappa$. It is located at $\overline{\boldsymbol{X}}-\boldsymbol{\kappa}$. The solid green lines indicate the $\bar{X}-1 \sigma$ values, which correspond to the $68 \%$ confidence interval if the statistics of the process are known. The dashed green line to the left of the solid green line is a lower bound for $68 \%$ confidence which includes the confidence interval on the mean. The dashed cyan line further to the left then shows the influence of applying the confidence interval of the variance instead of assuming that the computed variance is known with $100 \%$ confidence. The upper bound on the variance is used because that makes the variance a larger number, representing less certainty. 


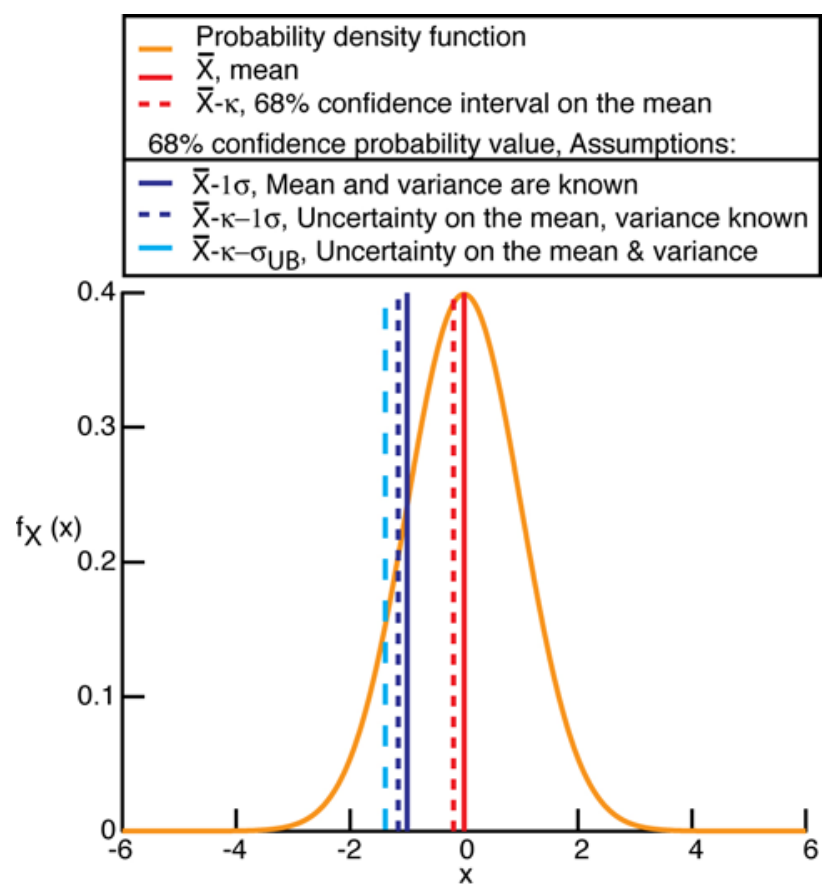

Figure 16. Confidence intervals on a normal distribution

The values for $\kappa$ and $\sigma$ are determined in the following two sections.

\section{A. Confidence interval on the mean}

Estimating the confidence interval for the mean of a normal distribution with a known variance uses the symmetry properties of the normal distribution and assumes use of a minimum width interval. The uncertainty on the mean is computed by transforming the process to a standardized random variable, assuming the maximum likelihood estimator for the mean is itself normal, and the probability density function of a Gaussian process ${ }^{6}$. This results in the uncertainty on the mean as expressed in Equation 4. The confidence interval bounds are determined by the uncertainty subtracted and added to the mean.

$$
\kappa=\frac{u_{\alpha / 2} \sigma}{\sqrt{n}}
$$

Equation 4

Note that this expression is strictly valid only for processes where the variance is known. The student's tdistribution and the sample variance are employed otherwise. Because there are a large number of degrees of freedom in this problem, the numerical results were identical to the results using the known variance. ${ }^{6}$

\section{B. Confidence interval on the variance}

The confidence interval for the variance is determined in a similar manner, but utilizes the chi-squared distribution function. Upper and lower bounds define the 100(1- $\alpha) \%$ confidence interval for the variance, ${ }^{6}$ Equation 5.

$$
P\left[\frac{(n-1) s^{2}}{\chi_{n-1, \alpha / 2}^{2}}<\sigma^{2}<\frac{(n-1) s^{2}}{\chi_{n-1,1-\alpha / 2}^{2}}\right]=1-\alpha
$$

The upper bound on the confidence interval for the variance is taken from Equation 5 and shown in Equation 6. 


$$
\left(\sigma_{U B}\right)^{2}=\frac{(n-1) s^{2}}{\chi_{n-1,1-\alpha / 2}^{2}}
$$

This study contained over 100,000 degrees of freedom in predicting the flutter dynamic pressure. As the number of degrees of freedom increases, the bounds squeeze in towards the point estimation of the variance. For an infinite number of degrees of freedom, the bounds converge to the point estimate. This corresponds to the situation where the variance of the process is known, and no uncertainty interval on the variance is required. Also, the chi-squared distribution becomes a Gaussian distribution as the number of samples goes to infinity, based on the central limit theorem. ${ }^{6}$ Equation 7 shows the behavior of the upper bound on the confidence interval as the number of degrees of freedom becomes large.

$$
\frac{(n-1)}{\chi_{n-1,1-\alpha / 2}^{2}} \rightarrow 1 \text {, so }\left(\sigma_{U B}\right)^{2} \rightarrow S^{2}
$$

Equation 7

\section{Gaussian confidence results}

Table 8 shows results utilizing point estimates of the mean and variance and incorporating the confidence intervals into the data. Each row in the table shows the lower bound on the flutter dynamic pressure for the percent confidence given in the second column. The fifth column shows the results when point estimates of the mean and variance are used; the sixth column shows the results when the confidence intervals on the mean and variance are included in the computation. For illustration, 100 degrees of freedom were assumed in calculating the bounds on the variance. Note that incorporating these additional uncertainties gives slightly more conservative flutter onset predictions.

Table 8. Uncertainty parameters and lower bound on flutter dynamic pressure; Gaussian theoretical model

\begin{tabular}{|c|c|c|c|c|c|}
\hline & & & & \multicolumn{2}{|c|}{$\begin{array}{c}\text { Lower Bound on flutter dynamic pressure } \\
\text { (psf) }\end{array}$} \\
\cline { 5 - 6 } $\begin{array}{c}\text { Sigma } \\
\text { Level } \\
(\gamma)\end{array}$ & $\begin{array}{c}\% \\
\text { Confidence }\end{array}$ & $\begin{array}{c}\text { Uncertainty } \\
\text { on the mean } \\
(\kappa)\end{array}$ & $\sigma_{\text {UpperBound }}^{2}$ & $\begin{array}{c}\text { Using point } \\
\text { estimates of mean } \\
\text { and variance }\end{array}$ & $\begin{array}{c}\text { Incorporating } \\
\text { confidence } \\
\text { intervals on the } \\
\text { mean and variance }\end{array}$ \\
\hline $1 / 2$ & 38 & 0.0029 & 3.62 & 76.21 & 76.17 \\
\hline 1 & 68 & 0.0058 & 3.9 & 75.29 & 75.14 \\
\hline 2 & 95 & 0.0113 & 4.51 & 73.46 & 70.86 \\
\hline 3 & 99.7 & 0.0168 & 5.41 & 71.63 & 70.13 \\
\hline
\end{tabular}

\section{Discussion}

The flutter margin method provides a quantitative method for utilizing frequency coalescence information available at subcritical test conditions. This work demonstrated that it is accurate for predicting the onset of classical coalescence flutter. This is true for application of the method both in a deterministic and a stochastic manner.

A fundamental question to consider is whether or not stochastic methods can be used to conduct flutter testing.

The stochastic methods provided good estimates of the flutter dynamic pressure, although slightly nonconservative. The non-conservatism is partially due to non-conservative modal trends, rather than the stochastic methods themselves. Applying these methods in an automated fashion removed the influence of engineering insight from data selection and interpretation. In real-time flutter testing, it is often this insight that builds the conservatism into the predictions. 
Traditionally, when conducting flutter testing in a wind tunnel, you do not have a large amount of data available at the subcritical conditions prior to proceeding to flutter. Using stochastic methods would require a change in test procedure, acquiring numerous sets of modal characteristics at each subcritical condition. Another possible source of multiple data sets is to utilize multiple sensors at each subcritical condition. This eases the requirement to repeat the same test condition, but the near real time processing requirements for each test condition increase.

For flutter testing where a Mach number-dynamic pressure boundary of flutter conditions are to be determined, stochastic methods may be suitable for determining the significance of the Mach number. If the subcritical data indicates that Mach number isn't a significant factor, the flutter dynamic pressure would be invariant. This statistical methodology could thus indicate the onset of compressible effects.

Using probability distribution models is a convenient approach because the stochastic behavior can be accomplished with only 2 or 3 parameters. However, the fits to the data are often not good. In this case, maximum likelihood parameter fits produced non-conservative gamma distribution models of the flutter dynamic pressure. To build conservatism into the model, a manual fit or another method of determining the coefficients needs to be used.

Calculating the Gaussian confidence intervals demonstrated that for large data sets, the statistical confidence in the distribution parameters is high, even when the model does not accurately represent the data. Meeker and Escobar ${ }^{2}$ state that uncertainty results from limited knowledge or limited data set. They separate it into uncertainty in model form and uncertainty in model parameters. "Uncertainty leads to uncertainty bounds- statistical confidence bounds quantify parameter uncertainty, but not model uncertainty." In this case, the confidence intervals didn't lend much insight to the problem. Rather than looking to the confidence intervals to determine the likelihood of flutter occurring, a measure of the goodness of the fit should be used to provide this insight.

A noticeable shortcoming of the treatments presented here is that discrete variables were not incorporated. This is important in considering the range of conditions to incorporate into the subcritical data set. While incorporating all available subcritical data seemed like a good idea at the time, it unduly biased the results, forcing the dynamic pressure estimates to be high. By definition, subcritical data occurs below the flutter condition. By including the $75 \mathrm{psf}$ data in all of the processes, there were only a minimal number of cases which produced flutter conditions below this value. A better approach would have incorporated knowledge of the number of times that the 75 psf data was post-flutter.

\section{Conclusions}

Experimental prediction of flutter onset using stochastic methods require a change in the process employed to conduct flutter testing. The methods evaluated here rely on numerous data points to quantify the uncertainty in modal characteristics at subcritical conditions. These methods are easily applied to in-hand historical data sets.

Stochastic treatment of all of the data from the PARTI test illustrated the extent to which engineering judgment was relied upon to build conservatism into the flutter predictions. While sample automated fits shown in the paper provided good predictions of the flutter condition, these predictions were not conservative.

One source of the non-conservatism is the definition and treatment of what constitutes the subcritical data set. Incorporating all available subcritical data forced the dynamic pressure estimates higher. A better approach would have incorporated knowledge of the number of times that the 75 psf data was post-flutter. Use of a discrete variable, indicating flutter or no flutter, might be useful for accomplishing this.

The theoretical distribution of the flutter dynamic pressure requires additional consideration. The goodness of the theoretical distribution is a major factor in whether the overall prediction of flutter onset is conservative. While the threshold gamma distribution parameters can be manually tuned to provide a 
conservative model, it is desirable to develop a parameter estimation process which includes this requirement.

Using stochastic methods, it was demonstrated that probability levels can be associated with different flutter speeds, both neglecting and accounting for the confidence intervals.

The methodology and conclusions of Poirel, Dunn and Porter are supported by analysis of the PARTI data. The methodology demonstrated an approach to handling uncertainty present in the data, in a manner that produced rational trends in the prediction of flutter onset. Both flutter margin methods were shown to produce good predictions of the flutter condition. The sensitivity of the quadratic fit extrapolation was also demonstrated by the percentage of cases that did not flutter. While the suggestion for development of confidence intervals was followed, the relevance of the confidence intervals for large data sets was shown to be negligibly small.

\section{References}

1 Pettit, C.L, "Uncertainty quantification in aeroelasticity: recent results and research challenges," Journal of Aircraft, Vol. 41, No. 5, September-October 2004.

2 Meeker, W.Q, and Escobar, L.A, "Statistical methods for probabilistic design,” IMS/ASA-SPES Spring Research Conference Paper 257-3, June 4, 1998.

3 Poirel, D., Dunn, S, and Porter, J, "Flutter-margin method accounting for modal parameter uncertainties,” Journal of Aircraft, Vol. 42, No. 5, September-October 2005.

4 Zimmerman, N.H, and Weissenburger, J.T, "Prediction of flutter onset speed based on flight testing at subcritical speeds,” Journal of Aircraft, Vol. 1, No. 4, July-August 1964.

5 McGowan, A.R.; Heeg, J.; Lake, R.C.: Results of Wind-Tunnel Testing from the Piezoelectric Aeroelastic Response Tailoring Investigation. AIAA $37^{\text {th }}$ Structures, Structural Dynamics, and Materials Conference, Salt Lake City, UT, April 1996.

$6 \quad$ Soong, T.T, "Probabilistic modeling and analysis in science and engineering,” John Wiley \& Sons, New York, 1981.

7 Abromowitz, M. and Stegun, I.A, Handbook of mathematical functions, U.S. Department of Commerce, Bureau of National Standards, 10 ${ }^{\text {th }}$ printing, December 1972.

8 NIST/SEMATECH e-Handbook of Statistical Methods, http://www.itl.nist.gov/div898/handbook/, March 7, 2007.

9 Pimentel, R.G, deChamplain, A, Kretscher, D, Stowe, R.A, and Harris, P.G, Generalized Formulation for Droplet Size Distribution in a Spray," $42^{\text {nd }}$ AIAA/ASME/SAE/ASEE Joint Propulsion Conference and Exhibit, Sacramento, California, July 10-12, 2006.

10 Bandourian, R, MacDonald, J, and Turley, R, “A comparison of parametric models of income distribution across countries and over time,” Brigham Young University, Department of Economics, June 2002.

11 Clark, J.B, Kim, M.C, and Kabe, A.M, "Statistical analysis of atmospheric flight gust loads analysis data,” Journal of Spacecraft and Rockets, Vol. 37, No.4, July-August 2000.

12 Heard, Atrid and Pensky, Marianna, "Confidence intervals for reliability and quantile functions with application to NASA space flight data,” IEEE Transactions on Reliability, Vol. 55, No. 4, December 2006.

13 Anonymous, “JMP statistics and graphics guide, release 6,” SAS Institute Inc, Cary, NC, 2005. 